Original

\title{
The Pathological Features of Alloxan Diabetes in Beagle Dogs
}

\author{
Dai Watanabe ${ }^{1,2}$, Hiromi Nakara ${ }^{1}$, Yuko Yamaguchi ${ }^{1}$, Keisuke Akagi ${ }^{1}$, \\ Toru Hoshiya ${ }^{1}$, Yoshikazu Nagashima ${ }^{1}$, Azusa Okaniwa ${ }^{1}$, and Hiroyasu Yoshikawa ${ }^{2}$ \\ ${ }^{1}$ Kannami Laboratory, Biology and Zoology (BOZO) Research Center Inc., 1308 Kuwabara-Sanbonmatsu, Kannami-cho, \\ Tagata-gun, Shizuoka-ken 419-0101, Japan \\ ${ }^{2}$ Department of Pathology, School of Veterinary Medicine and Animal Science, Kitasato University, Towada-shi, \\ Aomori-ken 034-8624, Japan
}

\begin{abstract}
Beagle dogs intravenously treated with alloxan monohydrate at $50 \mathrm{mg} / \mathrm{kg}$ and kept for 14 months were subjected to clinicopathological and histopathological examinations in order to characterize the development of diabetic changes. The beagle model of diabetes mellitus with alloxan in the present study was characterized by variegated conditions which markedly involved male animals. All the males in this study either died or were sacrificed in extremis up to Week 20, whereas all the females survived throughout the 14-month observation period. Determination of serum fructosamine level, and the glucose tolerance test proved useful in detecting diabetic signs. Decreased serum creatinine and increased total cholesterol levels, and elevation of alkaline phosphatase activity were important secondary changes; however no consistent signs of ketoacidosis were noticed in the persistently diabetic animals. In the present study, diestrus, as confirmed by estrous bleeding, may have been an important provoking factor in diabetic episodes in female dogs with impaired glucose tolerance. Pathological changes seen in the pancreas, liver, and kidneys were ascribed to direct effects of alloxan and the resulting derangement of carbohydrate metabolism. As early as Week 4 of treatment, ocular changes, especially development of cataract, were recorded as the major secondary complications ascribable to persistent hyperglycemia. There were no signs of structural complications, except for parenchymal atrophy and focal inflammatory changes in various organs. Secondary complications other than the cataract seemed to not easily occur in this animal model. (J Toxicol Pathol 2004; 17: 187-195)
\end{abstract}

Key words: diabetes mellitus, beagle dogs, alloxan, animal model, blood chemistry

\section{Introduction}

Aimed at clarifying the development of diabetic changes in an animal model, we carried out pathological observations on beagle dogs treated with alloxan. Numerous studies describing the effects of alloxan, one of the most classic diabetogens, on experimental animals, predominantly on mice and rats, have been published ${ }^{1}$. However, no studies to date have described the multiple pathological features as reported in the present study. In addition, the present study examined systemic effects due to toxic insults to the endocrine pancreas or carbohydrate metabolism in beagle dogs.

Received: 8 April 2004, Accepted: 24 July 2004

Mailing address: Dai Watanabe, Kannami Laboratory, Biology and Zoology (BOZO) Research Center Inc., 1308 Kuwabara-Sanbonmatsu, Kannami-cho, Tagata-gun, Shizuoka-ken 419-0101, Japan

TEL: 81-559-78-7851 FAX: 81-559-78-7898

E-mail: watanabe@bozo.co.jp

\section{Materials and Methods}

\section{Animals}

Ten beagle dogs ( 5 males and 5 females each, HRA beagle, Covance Research Products Inc. Va., U.S.A.) at 10 to 33 months of age were used in the present study. The body weight of the dogs ranged from 8.2 to $13.6 \mathrm{~kg}$ with a mean weight of $11 \mathrm{~kg}$ for males, and 6.9 to $10.2 \mathrm{~kg}$ with a mean weight of $8.3 \mathrm{~kg}$ for females just before treatment on Day 1 . They were housed in individual cages under routinely controlled condition $\left(18\right.$ to $26^{\circ} \mathrm{C}$; relative humidity: 30 to 80\%; lighting: 12 hours illumination per day), and provided a pellet diet (restricted feeding $300 \mathrm{~g} / \mathrm{animal} / \mathrm{day}$, DS-A, Oriental Yeast Co. Ltd., Tokyo, Japan), and tap water ad libitum. All experimental procedures of experimentation were done in accordance with the animal welfare guidelines of the Bozo Research Center Inc.

\section{Chemical, dose and treatment}

The treatment dose was determined after referring to the results of an exploratory study using beagle dogs. In the present study, 5\% alloxan monohydrate (Wako Pure 
Chemical Industries, Ltd., Osaka, Japan) dissolved in physiological saline at a dose level of $50 \mathrm{mg} / \mathrm{kg}$, was intravenously injected once to all animals in a fasting condition. Preparation and injection of the dosing solution were done as quickly as possible to avoid the effects of the denaturation of the dosing solution, because alloxan is reported to be extremely reactive and unstable ${ }^{2}$.

\section{In-life observation and examination}

All animals were observed daily for their general condition, and subjected to weekly body weight measurement from two weeks before treatment (Week-2) and throughout the study period. Hematological and blood chemical examinations, urinalysis, and ophthalmological evaluations including electroretinography were performed twice in Weeks -2 and -1 (pretreatment period), once on Day 1 of the study ( 5 hours after treatment), and then, every two weeks up to Week 6, and thereafter monthly up to Month 14. Hematological and blood chemical examinations were performed using blood samples collected from the cephalic vein with standard laboratory equipment and assay techniques validated for the laboratory. Collection of blood samples were performed in the morning after deprivation of food for about 16 hours. In addition, hematological and blood chemical examinations were performed on all the animals in the morning on Day 2 without previous fasting. The following hematological parameters were determined using a Coulter counter T 890 (Beckman-Coulter, Inc.Calif. USA): RBC, hematocrit, hemoglobin, $\mathrm{MCH}, \mathrm{MCHC}, \mathrm{MCV}$, WBC and platelet count. Blood chemical parameters included ASAT, ALAT, ALP, total cholesterol, triglyceride, phospholipids, total bilirubin, glucose, BUN, creatinine, sodium, potassium, chloride, phosphorus and total protein. Clinical Laboratory System TBA-120FR (Toshiba, Co. Tokyo, Japan) was employed to determine these blood chemical parameters. Previous researchers have been treating their diabetic dogs with insulin to prolong survival $^{3,4}$, however, no such management measures were undertaken according to the objectives of the present study.

In addition to the routine examination items described above, the following blood and urine glucose-related parameters were examined for the purpose of diagnosing the diabetes, quantification of urine glucose and serum fructosamine, as well as an oral glucose tolerance test. The quantification of urine glucose was performed because glucosuria is known as the most important index of glucose intolerance. Glucosuria seems to occur when serum concentrations of glucose exceed the renal threshold (180 $\mathrm{mg} / \mathrm{dL})^{5}$. The methods and reagents used in the glucose tolerance test, and in the determination of serum fructosamine have been previously described ${ }^{6}$. As described in that report, gavage administration of $50 \%$ glucose solution at $5 \mathrm{~mL} / \mathrm{kg}$ per body weight was performed under fasting conditions, and a 180-minute level was evaluated in the oral glucose tolerance test. The biochemical criteria used in the diagnosis of diabetes are summarized in Table 1.

\section{Postmortem examination}

All male dogs found dead or euthanatized in moribund condition were immediately subjected to autopsy, organ weight measurement and sampling of histopathological specimens as soon as possible. All female dogs survived to the end of the observation period in Month 14 and were sacrificed by blood withdrawal under deep anesthesia with pentobarbital, and subjected to further examinations in a similar manner to males. Histopathological examinations of major organs and tissues were conducted according to routine procedures. In addition, immunohistochemical staining to demonstrate the presence of insulin-secreting $\beta$ cells using an anti-insulin antibody (Anti-insulin antibodies and Auto Probe II HRP/DAB Kits, Biomeda Corp. California, USA) was performed on the pancreas of five male animals. Whole eyes were immersed in Bouin's fixative for about three to four hours, then dissected and immersed in buffered $10 \%$ formalin. Sections of the eyes and pancreas were subjected to the Periodic acid-Schiff' (PAS) method.

\section{Evaluation of data}

All numerical data generated during in-life observations, and in postmortem examinations were expressed as actual values, and percentile changes from those of Week -1 (pretreatment baseline values). All data were then evaluated on an individual basis by comparison with baseline data.

\section{Results}

The overall summary of results for in-life observations and examinations is shown in Table 2.

\section{Findings of clinical observation and serum glucose related-parameters \\ Male and female dogs observed in this study exhibited} illnesses of a distinctive nature different from each other: acute to subacute disease with a fatal course in males, and a chronic course of disease with varying degrees without fatality in females.

Male dogs (Nos. 2, 3, 4, 5) took a fatal course exhibiting marked diabetic signs and died or were sacrificed in extremis on Days 76, 122, 135, and 51, and one animal (No.1) died on Day 35. The former four male animals similarly exhibited, irrespective of course duration, marked polyuria and polydipsia, glucosuria and hyperglycemia after Day 1 (from Week 2 in one animal, No.4), and an increased fructosamine concentration during and after Week 2. Glucosuria was the earliest and most remarkable sign, with a daily total excretion of glucose reaching approximately 10 to 60 times that of baseline values. The daily total excretion of glucose reached a maximum (about several hundred thousand times as high as baseline values) in Week 2, and the value remained unchanged afterward until death. Prior to the observations of these signs, one male dog (No.4) was found to be hypoglycemic $(68 \mathrm{mg} / \mathrm{dL}) 24$ hours after treatment. 
Table 1. Biochemical Criteria Used in the Diagnosis of Diabetes in Dogs

\begin{tabular}{llccc}
\hline Items with units & & Reference interval and means ${ }^{4}$ & Diabetic \\
\hline Fasting serum glucose & $\mathrm{mg} / \mathrm{dL}$ & $88-112$, & 97 & $>130$ \\
Urine glucose & $\mathrm{mg} / \mathrm{dL}$ & $9-21$, & 15 & $>40$ \\
Serum fructosamine & $\mu \mathrm{mol} / \mathrm{L}$ & $93-123$, & 108 & $>141$ \\
Serum glucose & $\mathrm{mg} / \mathrm{dL}$ & $87-105$, & 95 & $>140$ \\
(glucose tolerance test) & & \multicolumn{2}{c}{$(180$ min. value $)$} & $(180 \mathrm{~min})$. \\
\hline
\end{tabular}

CRITERIA BASIS IN THE DIAGNOSIS OF DIABETES. Urine glucose: more than twice the upper limit of the reference interval. Other parameters: exceeding $15 \%$ or more the upper limits of the reference interval.

Table 2. Summary of Clinical Observation and Clinicopathological Examination

\begin{tabular}{|c|c|c|c|c|c|c|c|c|c|c|c|}
\hline & Animal No. & 1 & 2 & 3 & 4 & 5 & 6 & 7 & 8 & 9 & 10 \\
\hline 우한 & and Age (months) & $\begin{array}{c}\delta \\
33\end{array}$ & $\begin{array}{c}\pi \\
33\end{array}$ & $\begin{array}{c}\delta \\
19\end{array}$ & $\begin{array}{c}\sigma \\
11\end{array}$ & $\begin{array}{c}\pi \\
10\end{array}$ & $\begin{array}{l}\text { 우 } \\
11\end{array}$ & $\begin{array}{l}\text { 우 } \\
11\end{array}$ & $\begin{array}{l}\text { 우 } \\
11\end{array}$ & $\begin{array}{l}\text { 우 } \\
11\end{array}$ & $\begin{array}{l}\text { 우 } \\
11\end{array}$ \\
\hline & Hypoglycemia & & & & $\begin{array}{c}0 \\
68\end{array}$ & & & & $\begin{array}{c}\bigcirc \\
57\end{array}$ & $\begin{array}{l}0 \\
57\end{array}$ & $\begin{array}{l}0 \\
49\end{array}$ \\
\hline INITIAL AND & Glucosuria & & & & & & & & $\begin{array}{c}0 \\
433\end{array}$ & & \\
\hline CHANGES & Hyperglycemia & & & & & & & & $\begin{array}{c}0 \\
144\end{array}$ & & \\
\hline & Increased BUN & & & & & $\begin{array}{c}0 \\
\times 2.7\end{array}$ & $\begin{array}{c}0 \\
\times 1.8\end{array}$ & & $\begin{array}{c}0 \\
\times 2.0\end{array}$ & & $\begin{array}{c}0 \\
\times 2.8\end{array}$ \\
\hline & Polyuria & & 0 & 0 & 0 & 0 & & 0 & & & \\
\hline & Polydipsia & & 0 & 0 & 0 & 0 & & 0 & & & \\
\hline & Body Weight Loss & & $\begin{array}{c}0 \\
53 \%\end{array}$ & $\begin{array}{c}0 \\
45 \%\end{array}$ & $\begin{array}{c}0 \\
46 \%\end{array}$ & $\begin{array}{c}0 \\
43 \%\end{array}$ & & 0 & & & \\
\hline & Glucosuria & 0 & 0 & 0 & 0 & 0 & 0 & 0 & 0 & 0 & 0 \\
\hline & Hyperglycemia & & 0 & 0 & 0 & 0 & & 0 & & & \\
\hline & Elevated Fructosamine & 0 & 0 & 0 & 0 & 0 & O & 0 & 0 & $\mathrm{O}$ & 0 \\
\hline$\underline{\text { PERSISTENT }}$ & Impaired Glucose Tolerance & 0 & 0 & 0 & 0 & 0 & 0 & 0 & 0 & 0 & 0 \\
\hline CHANGES & Decreased Creatinine & & $\begin{array}{c}\bigcirc \\
\times 0.2\end{array}$ & $\begin{array}{c}0 \\
\times 0.2\end{array}$ & $\begin{array}{c}\bigcirc \\
\times 0.4\end{array}$ & $\begin{array}{c}\bigcirc \\
\times 0.5\end{array}$ & & $\begin{array}{c}\bigcirc \\
\times 0.4\end{array}$ & & & \\
\hline & Elevated SALP & & $\begin{array}{c}0 \\
\times 4.6\end{array}$ & $\begin{array}{c}0 \\
\times 3.5\end{array}$ & $\begin{array}{c}0 \\
\times 2.5\end{array}$ & $\begin{array}{c}0 \\
\times 1.9\end{array}$ & & $\begin{array}{c}0 \\
\times 2.1\end{array}$ & & & \\
\hline & Increased T. Cholesterol & & $\begin{array}{c}0 \\
\times 1.6\end{array}$ & $\begin{array}{c}0 \\
\times 2.0\end{array}$ & $\begin{array}{c}0 \\
\times 1.9\end{array}$ & $\begin{array}{c}0 \\
\times 1.9\end{array}$ & & $\begin{array}{c}0 \\
\times 1.9\end{array}$ & & & \\
\hline & Increased Platelet Count & & $\begin{array}{c}0 \\
\times 2.03 \\
\end{array}$ & $\begin{array}{c}0 \\
\times 2.05 \\
\end{array}$ & $\begin{array}{c}0 \\
\times 2.08 \\
\end{array}$ & & & $\begin{array}{c}0 \\
\times 1.69 \\
\end{array}$ & & & \\
\hline & Died & D35 & & & & & & & & & \\
\hline OUTCOME & Sacrificed in extremis & & D76 & D122 & D135 & D51 & & & & & \\
\hline & Scheduled Sacrifice in M14 & & & & & & $\mathrm{O}$ & 0 & 0 & 0 & 0 \\
\hline
\end{tabular}

Initial and temporary changes in serum and urine glucose concentration were expressed as actual measurements (unit: $\mathrm{mg} / \mathrm{dL}$ ). Body weight loss: Difference between initial and terminal body weights (\%). Values of blood chemical and hematological examinations: Comparison between baseline and temporary/terminal values (times vs. baseline value). D: Day

Although these male dogs consumed all diet given, all dogs appeared to be emaciated, exhibiting a marked decrease in body weight from 43 to $53 \%$ of baseline values, and were observed in a sitting or lateral position and lying down before death. Urinalysis performed using reagent strips revealed a slightly decreased alkalinity during and after Month 2, and ketone body-positive reaction during and after Month 1, which was always found to be slight and changeable. In one exceptional case (No.1), glucose intolerance was recorded in Week 1, glucosuria on Day 1, and an increased fructosamine concentration in Weeks 2 and 4. No hyperglycemia, however, was recorded in this animal.

Female dogs exhibited no severe signs of adverse reaction to treatment during the 14-month observation period. One female (No.7) continuously exhibited signs of diabetes such as glucosuria, which was recorded on Day 1 (onset of the sign, hereafter the same), glucose intolerance in Week 1, increased fructosamine concentration in Week 2, and hyperglycemia in Month 4. Changes in the general condition of this diabetic female dog were mild, and the 
Table 3. Sequential Relationship between Estrous Hemorrhage and Temporal Elevation of Serum Fructosamine Level

\begin{tabular}{lccccc}
\hline & Animal No. & No. 6 & No. 7 & No. 8 & No. 9 \\
\hline Estrous hemorrhage & Weeks & Weeks & Weeks & Weeks & Weeks \\
& $28-30$ & $30-32$ & $16-18,56$ & $32-34$ & Week 37 \\
\hline Elevation of fructosamine level & Week 37 & Week 37 & Weeks 23, 37, 59 $29,34,37$ \\
\hline
\end{tabular}

Changes in fructosamine level shown for Nos. 7 and 10 in this Table are also shown in Figs. 2-3) and 2-4).

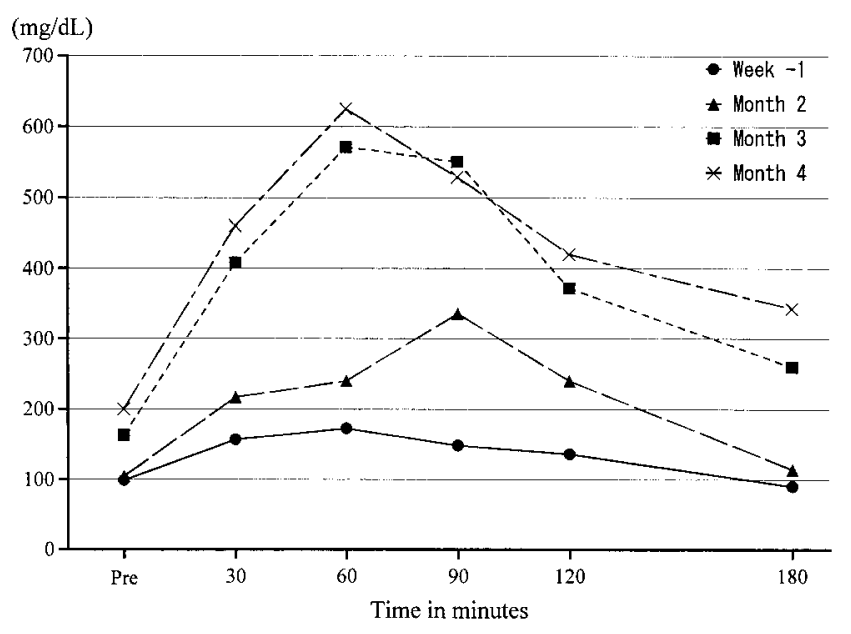

Fig. 1. Aggravation of glucose tolerance during the course in a persistently diabetic but survived female. Maximum serum glucose level (60 minutes after glucose loading) is close to but somewhat lower than the renal-threshold (about $180 \mathrm{mg} / \mathrm{dL}$ ) in pretreatment baseline examination. Extent of impaired glucose tolerance reached maximum during the first four months. Pre: fasting serum glucose (baseline value). Time in minutes: time after loading with oral glucose loading $(50 \%$ solution, $5 \mathrm{~mL} / \mathrm{kg}$ ).

decrease in body weight during the 14-month observation period remained at approximately $34 \%$. The extent of impaired glucose tolerance apparently worsened during the first four months as shown in Fig. 1. Results of urinalysis were similar to those recorded in male animals. Four other female dogs were sporadically diabetic, exhibiting glucosuria, impaired glucose tolerance, and increased fructosamine concentration following hypoglycemia (49-57 $\mathrm{mg} / \mathrm{dL}$ ) five and/or 24 hours after treatment in three animals (Nos. 8, 9, 10). Variations in these parameters were mild, and no noticeable effects on serum glucose level, body weight, and on the general condition were recorded. Among the variations in these diabetic parameters, changes in fructosamine concentration always followed changes in glucose intolerance or glucosuria. These females exhibited estrous hemorrhage prior to the diabetic episodes. The sequential relationship between estrous hemorrhage and temporal elevation of serum fructosamine level is shown in Table 3. As seen in the Table, elevation of serum fructosamine level followed estrous hemorrhage by about seven weeks. Sequential changes in diabetic-related parameters in representative cases are shown in Figs. 2-1) to 2-4).

\section{Other blood chemical and hematological findings}

Consistent variation was recorded for serum creatinine, serum alkaline phosphatase (SALP), and serum total cholesterol concentrations exclusively in five cases of persistent diabetes ( 4 males and 1 female). The serum creatinine concentration (range in Week -1 : $0.50-0.64 \mathrm{mg} /$ $\mathrm{dL}$ with a mean $0.56 \mathrm{mg} / \mathrm{dL}$ ) decreased during Weeks 2 (No.3), 4 (No.2, 4, 5) or Month 3 (No.7), and gradually worsened in parallel with the course of illness, reaching values ranging from $0.13-0.28 \mathrm{mg} / \mathrm{dL}$ with a mean of 0.18 $\mathrm{mg} / \mathrm{dL}$ in the examination prior to death or sacrifice. No consistent variation was recorded for other parameters relating to renal function in these animals. Transient and slight increases in blood urea nitrogen ranging from 1.6 to 2.8 times that of baseline values were recorded; however, the variations were by no means simultaneously recorded with the changes in creatinine levels. Elevation of SALP activity (about 1.5 times of baseline value) was recorded in Week 2 (Nos. 3 and 4) at the earliest, and at the latest in Month 3 (No.7), and the value gradually increased to 1.9-4.6 times that of baseline values in the last examination prior to death or sacrifice. An increase in serum total cholesterol concentration which ranged from 1.6-2.0 times that of baseline value was recorded in examinations between Week 4 and Month 4. Then it remained unchanged throughout the remainder of the study. In parallel with the increase in serum total cholesterol, a slight increase in serum phospholipids was recorded.

In hematological examinations, an increase in platelet count was recorded in four persistently diabetic cases ( 3 males and 1 female) that survived more than three months. The change became obvious after examination in Week 4 in three males, and in Month 8 in one female, and ranged from 1.69-2.08 times that of baseline values in the last examination prior to death or sacrifice.

\section{Ophthalmological findings}

The only findings ascribable to the treatment were cataractous changes appearing as lenticular subepithelial and cortical opacities in the equator of both eyes. All male dogs except one that died on Day 35, and one female dog, which was the only one animal with persistent diabetic signs in the female group, exhibited the above-mentioned clinical signs as early as in Week 4 at the earliest, and in Month 3 at the 

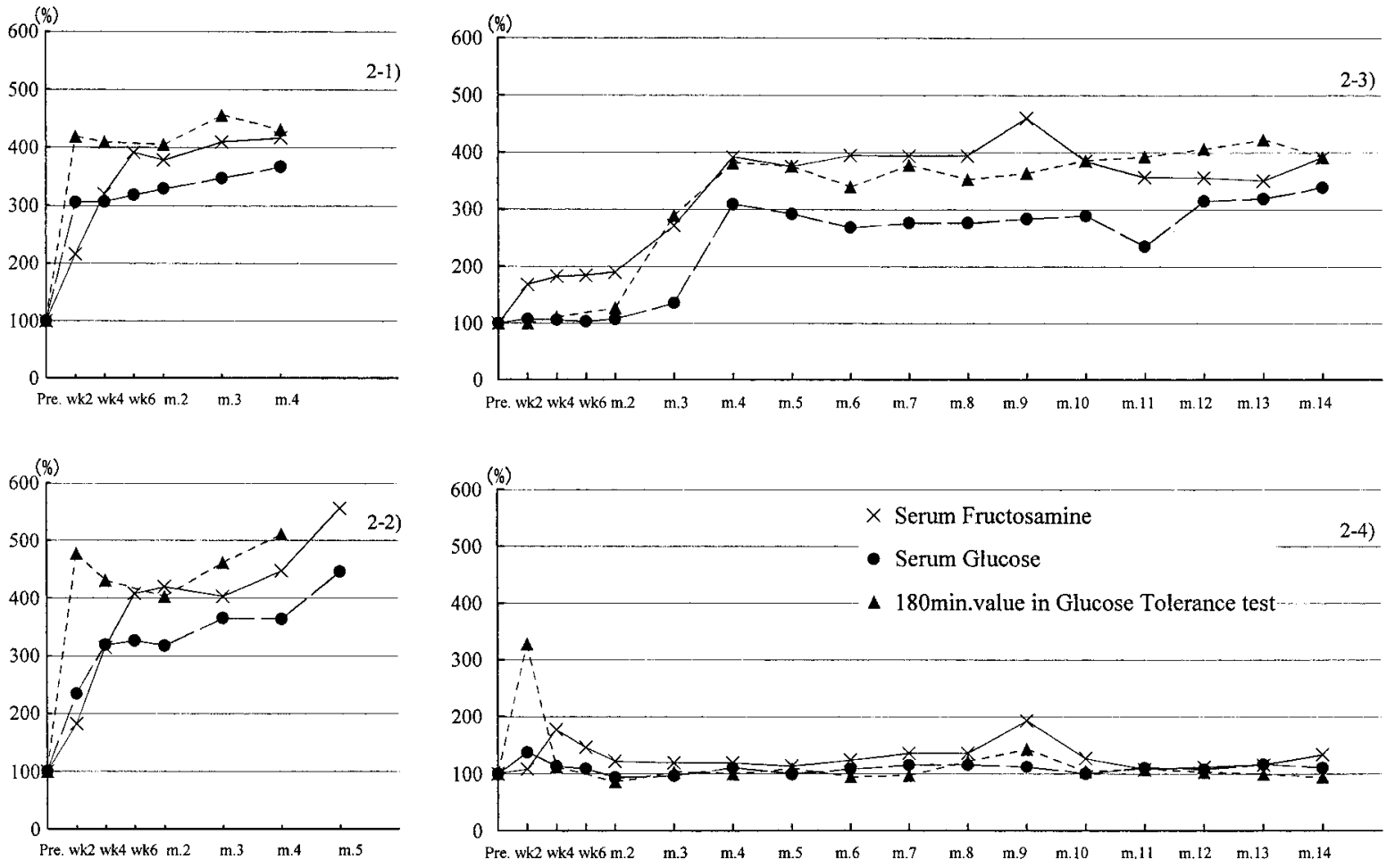

Fig. 2-1) to 2-4).

Sequential changes in fasting serum glucose, serum fructosamine, and 180 minutes value in oral glucose tolerance test in cases with various course. Data expressed as percentile change from baseline data (pre.). 2-1) and 2-2): acute and persistently diabetic fatal males. (Nos.3 and 4). 2-3): mild and persistently diabetic survived female. (No.7). Up to Month 2 only elevation of fructosamine is marked. 2-4): mild and sporadically diabetic female. (No.10). Throughout the observation period, only occasional elevation of fructosamine level is noticed, except for temporary impairment of glucose tolerance in Week 2.

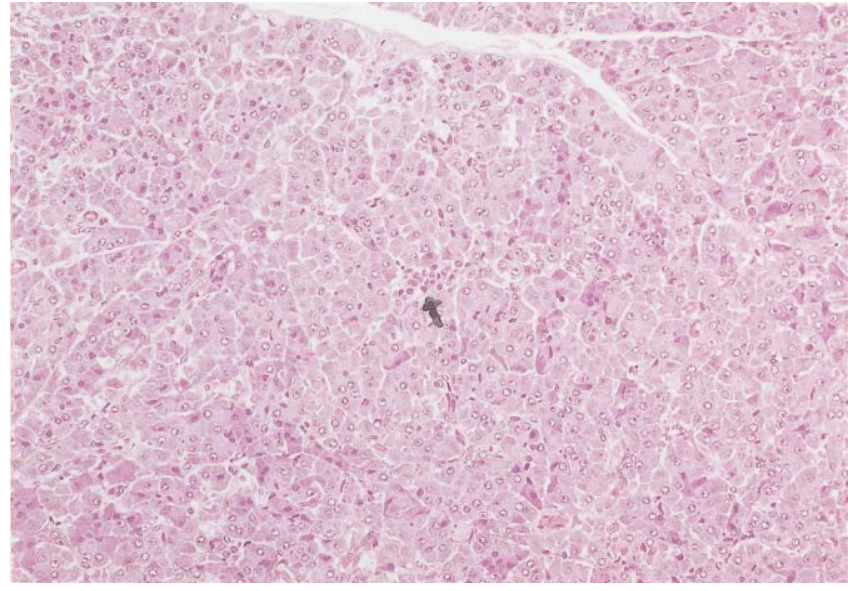

Fig. 3. Markedly atrophied Islets of Langerhans showing infiltration of lymphocytes (center of the figure, indicated by an arrow) showing typical view of so-called "insulitis" (No.2). Cells of exocrine pancreas also atrophied and contain less zymogen granules. HE, $\times 170$.

latest. The opaque lesion extended over the anterioposterior cortex of both lens. In all other aspects including the electroretinographic recordings, there were no abnormal findings throughout the observation period.

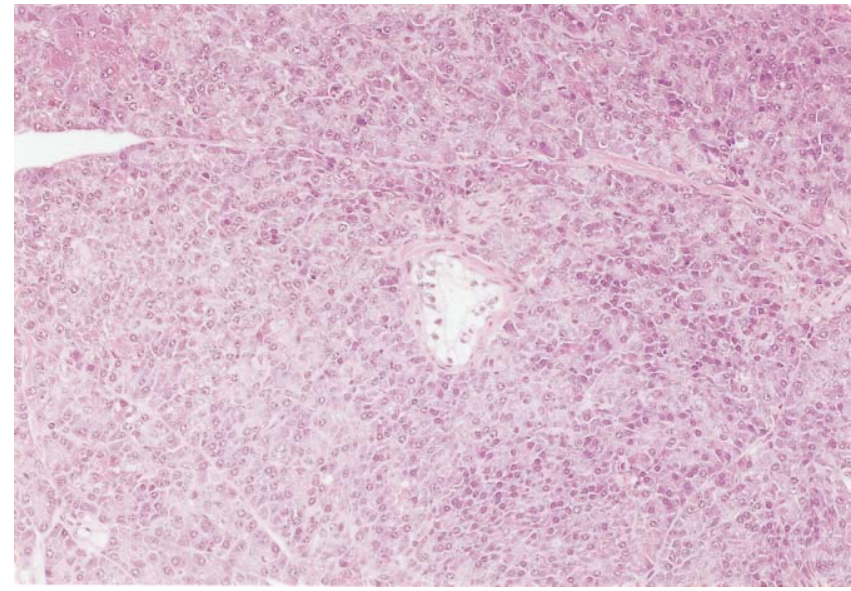

Fig. 4. Pancreas showing highly vacuolated and swollen ductal epithelia (No.2). Entire acinar cells exhibited marked atrophy with scanty zymogen granules. HE, $\times 170$.

\section{Pathological findings}

Autopsy revealed remarkable emaciation with slender muscles and no discernible subcutaneous and visceral adipose tissue in all persistently diabetic cases (4 males and 1 female). The relative weights for such organs as the brain, 


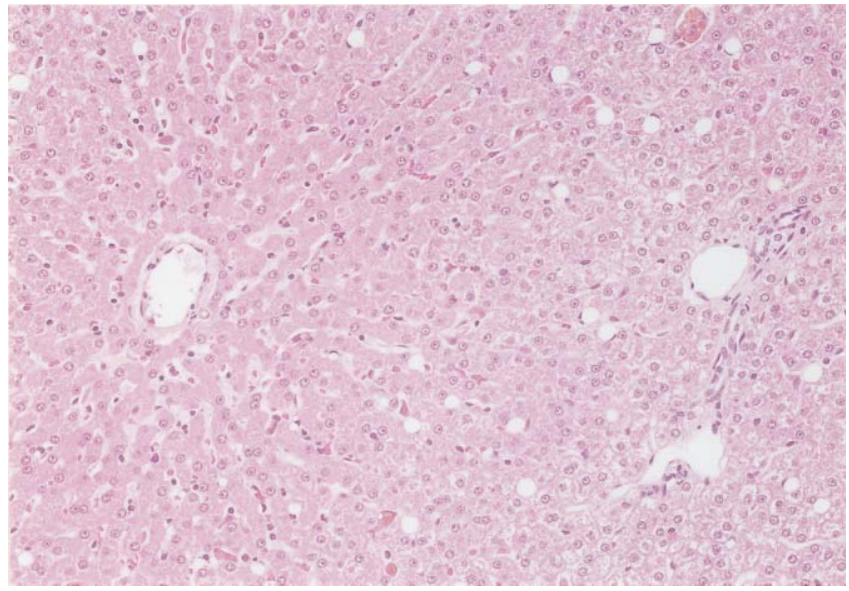

Fig. 5. Typical picture of liver changes in dogs took fatal course (No.2). Centro- and mid-lobular hepatocytes markedly atrophied and evenly stained. Vacuolated Ito cells are seen in the peripheral zone. HE, $\times 170$.

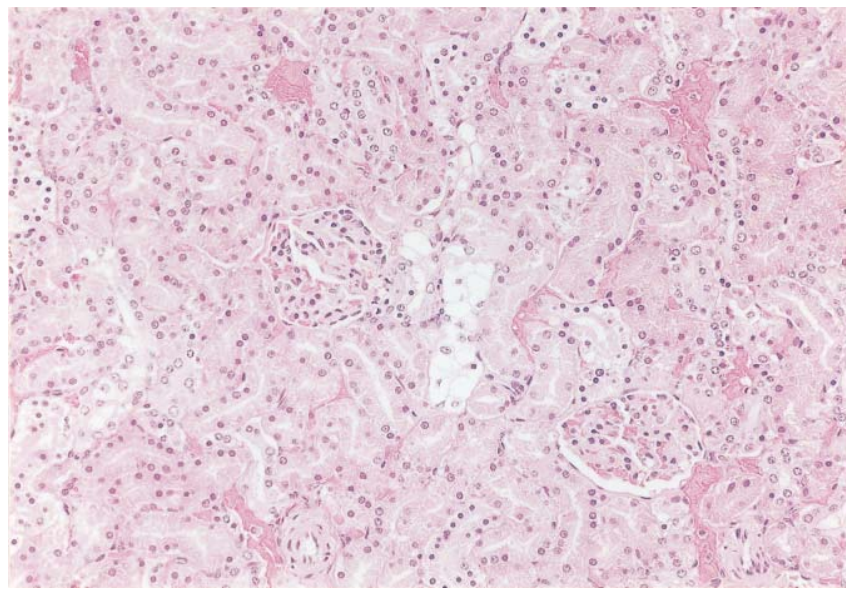

Fig. 7. Kidney showing changes similar to "Armanni-Ebstein Lesion' (No.4). Vacuolation was thought to represent the sites of glycogen deposit. HE, $\times 170$.

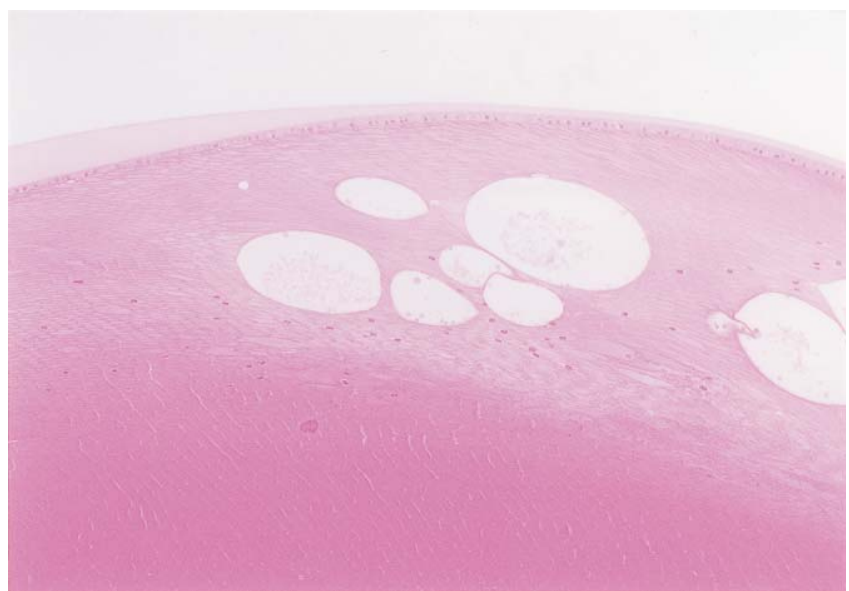

Fig. 9. Higher magnification of lenticular degeneration shown in Fig. 8 (No.3). Swelling of lenticular fibers extended, and the gaps contain flocculent material. HE, $\times 80$.

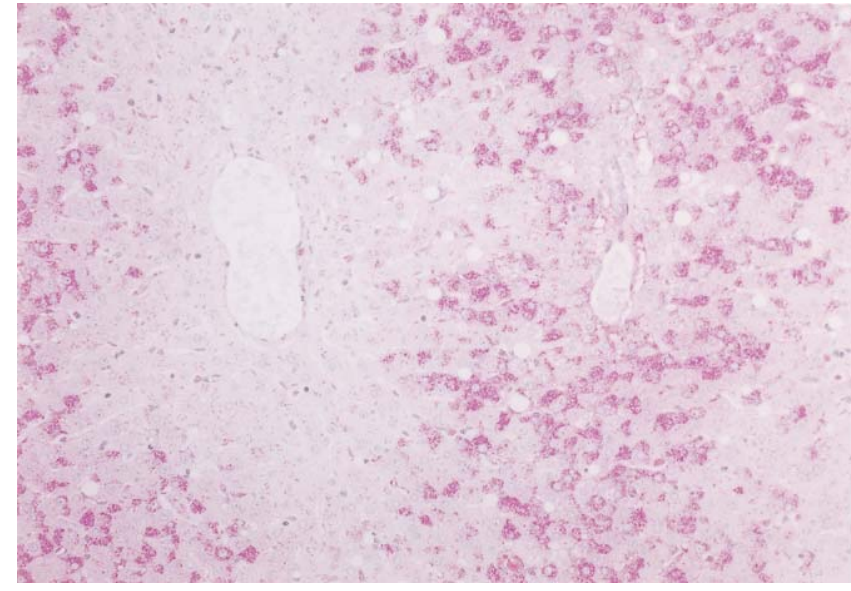

Fig. 6. Reversed pattern for glycogen deposition in hepatic lobules resulted from glycogen depletion in centrilobular hepatocytes (No.2). PAS, $\times 170$.

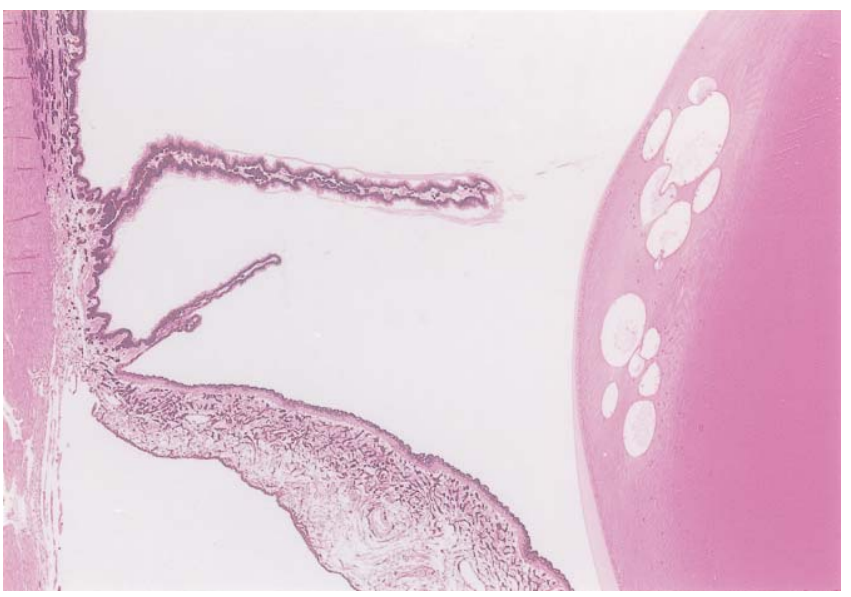

Fig. 8. Region adjacent to equator of lens (No.3). Gaps in varying size are conspicuous. HE, $\times 30$.

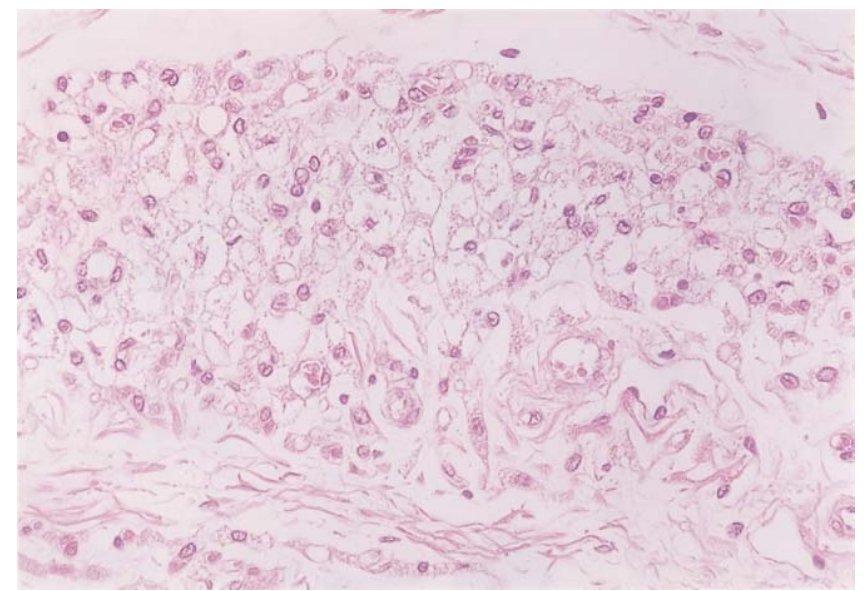

Fig. 10. Abdominal adipose tissue (No.4). Adipose cells lost their original aspect, and their cytoplasm exhibited gelatinous appearance. HE, $\times 340$. 
Table 4. Major histopathological findings

\begin{tabular}{|c|c|c|c|c|c|c|c|c|c|c|}
\hline \multirow[t]{2}{*}{ Findings } & \multicolumn{9}{|c|}{ Animal number } & \multirow[b]{2}{*}{10} \\
\hline & 1 & 2 & 3 & 4 & 5 & 6 & 7 & 8 & 9 & \\
\hline \multicolumn{11}{|l|}{ Pancreas } \\
\hline Atrophy of islets & 2 & 4 & 4 & 3 & 3 & 2 & 4 & 2 & - & - \\
\hline Decreased zymogen granules in acinar cells & 3 & 3 & 2 & 3 & 1 & - & 2 & - & - & - \\
\hline Paleness and hypertrophy of ductal epithelia & - & 2 & 1 & 2 & 1 & - & - & - & - & - \\
\hline Immunohistochemical staining for insulin-secreting $\beta$-cells & $1 *$ & - & - & - & - & / & / & / & / & / \\
\hline \multicolumn{11}{|l|}{ Liver } \\
\hline Atrophy / decrease in glycogen granules in hepatocytes & 3 & 2 & 2 & 3 & 3 & - & 1 & - & - & - \\
\hline Vacuolation of Ito cells in peripheral zone & 1 & 2 & 2 & - & - & - & - & - & - & - \\
\hline Vacuolation of bile ductal epithelia & - & 1 & 2 & 2 & 1 & - & 2 & - & - & - \\
\hline \multicolumn{11}{|l|}{ Kidney } \\
\hline Armanni-Ebstein change-like vaculolation & - & - & - & 1 & - & - & - & - & - & - \\
\hline Atrophy of adipose tissue & - & 3 & 3 & 3 & - & - & - & - & - & - \\
\hline \multicolumn{11}{|l|}{ Eye } \\
\hline Degeneration of lenticular fiber & - & 3 & 2 & 2 & 2 & - & 2 & - & - & - \\
\hline Keratitis & - & - & 1 & - & - & - & - & - & - & - \\
\hline \multicolumn{11}{|l|}{ Skin (abdominal) } \\
\hline Atrophy of adipose tissue & - & 4 & 4 & 4 & - & - & - & - & - & - \\
\hline \multicolumn{11}{|l|}{ Skeletal muscle (femoral) } \\
\hline Atrophy & - & 3 & 3 & 4 & - & - & - & - & - & - \\
\hline \multicolumn{11}{|l|}{ Lymph node (mesenteric, submandibular) } \\
\hline Atrophy & 1 & - & 2 & 3 & 1 & - & - & - & - & - \\
\hline Lymphadenitis & - & 2 & - & - & - & - & - & - & - & - \\
\hline \multicolumn{11}{|l|}{ Lung } \\
\hline Alveolar cell infiltration & - & - & - & 3 & - & - & - & - & - & - \\
\hline \multicolumn{11}{|l|}{ Thymus } \\
\hline Atrophy & 4 & 3 & 3 & 3 & 3 & 2 & 3 & - & 1 & 2 \\
\hline \multicolumn{11}{|l|}{ Urinary bladder } \\
\hline Cystitis & - & - & 2 & - & - & - & - & - & - & - \\
\hline
\end{tabular}

-: no remarkable changes, 1 : slight, 2: mild, 3: moderate, 4: severe, *: slightly positive, /: not examined

lungs, liver, kidneys, and adrenals were found to be higher than normal.

Major histopathological changes were observed almost exclusively in the pancreas, liver, kidneys, and eyes of persistently diabetic animals. A summary of histopathological findings is shown in Table 4. Mild to severe atrophy of the islets of Langerhans in the pancreas was found to be the most striking feature in these animals (Fig. 3). Some atrophied islets of Langerhans were infiltrated with lymphocytes. No discernible islets were found in severely and persistently involved cases in routine $\mathrm{H} \& \mathrm{E}$ preparations as well as in immunohistochemical preparations stained for insulin. Similar but mild changes were seen in the pancreas of one male (No.1) that died on Day 35, and in two females (Nos. 6 and 8) that only sporadically exhibited diabetic signs. In addition, paleness and hypertrophy of the ductal epithelia and a decrease in zymogen granules in acinar cells of the exocrine pancreas were observed (Fig. 4). No PAS-positive material was observed in the cytoplasm of hypertrophic ductal epithelia. The islets of Langerhans appeared normal in two females (Nos. 9 and 10), both of which exhibited only sporadic signs of diabetes. In the liver, a marked decrease in glycogen granules in atrophic hepatocytes, especially in those of the centrilobular and mid-zonal areas was marked and resulted in a reversed pattern of glycogen distribution in the hepatic lobulus in animals with persistent diabetes. At the same time, swollen and vacuolated Ito cells were conspicuous mainly in the peripheral zone in two males (Nos. 2 and 3) with persistent diabetes and the one male which died on Day 35 (Figs. 5, 6). In addition, epithelial vacuolation was observed in the biliary ducts and gall bladder. In the kidneys of one male dog (No.4), vacuolation of epithelial cells in the proximal straight renal tubules, similar to the so-called "Armanni-Ebstein Lesion" was observed (Fig. 7). In the eyes, lenticular degeneration in mild to moderate degrees was observed in persistently diabetic animals. The lesion consisted of swelling of the lenticular fibers and gap formation among the equatorial portion of the lesions (Fig. 8). The gaps and cavities among swollen lenticular fibers contained flocculent material and no PAS-positive material (Fig. 9). In a severely affected case (No.2), the change extended into superficial fibers of both the anterior and posterior poles. The above-mentioned lesion corresponded to the lenticular opacity seen in the ophthalmoscopic examination. Adipose tissue located throughout the animal's body was atrophic, and the cytoplasm of adipose cells exhibited a gelatinous appearance (Fig. 10). 
Furthermore, atrophy of parenchymal cells and focal inflammatory changes were widely observed in various organs and tissues in animals that were diabetic and took a fatal course. In addition, atrophy of the femoral skeletal muscle in moderate to severe degrees was seen in these animals. In the male that died on Day 35, no remarkable histopathological changes sufficient to explain the cause of death were found in all the organs and tissues examined.

\section{Discussion}

In the present study, diabetic conditions of varying degrees, lethal, persistent, and latent, were produced in beagle dogs treated with alloxan. In addition to known factors relating to pathogenesis of diabetes, individual and/or sex difference in susceptivities may be important. With respect to sex differences in dogs, female animals appeared to be less sensitive to alloxan treatment in the present study. No studies to date examining alloxan treatment in dogs and/ or other experimental animals have distinguished any sex differences with respect to alloxan sensitivity. Transient hypoglycemia, hyperglycemia, and glucosuria recorded in $\operatorname{dog} 5$ and/or 24 hours after treatment on Day 1 in one male (No.4) and in three females (Nos. 8, 9, 10) are likely to have corresponded to the three phase changes in blood glucose following single cytotoxic doses of alloxan and streptozotocin reported in rodents ${ }^{1,2}$. In the present study, similar initial changes may have actually occurred in all treated animals; however, many changes were thought not to have been detected because of their transient nature and the timing of the examinations. One male dog that died on Day 35 presented with characteristic features of illness different from those seen in other fatal cases. This animal exhibited neither persistent hyperglycemia nor any other associated secondary changes throughout the course of treatment; however his general condition gradually worsened, taking on a prone position before death. Generally, acute death within the first two days following alloxan treatment has been ascribed to severe hypoglycemia ${ }^{7}$ and death within one week following treatment has been ascribed to renal failure ${ }^{7,8}$, however, no definite evidence for renal involvement and/or hypoglycemia were recorded in this acutely ill dog. Therefore, the causes of deterioration in this animal's general condition and subsequent death were not established.

Four out of five males and one out of five females exhibited typical signs similar to naturally occurring diabetes mellitus in $\operatorname{dogs}^{9}$. When examining the sequence and relationship among diabetic-related blood chemical parameters in one female (No.7) which took a chronic course, as shown in Fig. 2-3), elevation of serum fructosamine levels, glucose intolerance and marked glucosuria were the first detectable signs during the first two months. In Month 3, impairment of oral glucose tolerance became apparent. A marked elevation in fasting serum glucose level was recorded after Month 4. Marked glucosuria and an elevation in serum fructosamine level were the most remarkable changes recorded throughout the course of observations in this case.

According to Duncan et al., resorption is complete in the proximal tubules when serum concentrations of glucose are less than $180 \mathrm{mg} / \mathrm{dL}$ in the $\mathrm{dog}$, whereas higher values are known to exceed the transport maximum resulting in glucosuria ${ }^{5}$. Accordingly, the extent of glucosuria is an indirect expression of the level of hyperglycemia. The extent of glucosuria and serum fructosamine levels described above almost always paralleled one another; however, the elevation of the latter parameter occurred about two weeks later. This would be due to the fact that fructosamine represents glycated serum protein, and its measurement reflects the average plasma glucose concentration (time-averaged indices) over one to three weeks ${ }^{10}$. Consequently the examination of urine glucose, glucose tolerance, and serum fructosamine are of great importance in the research of possible effects of xenobiotic compounds on carbohydrate metabolism.

The persistent diabetic changes described above accompanied various secondary changes in blood chemical and hematological parameters such as a decrease in serum creatinine concentration, elevation of SALP levels, an increase in serum total cholesterol concentration, and an increase in platelet count (thrombocytosis). The abovementioned changes in blood chemical and hematological parameters except changes in serum total cholesterol steadily worsened throughout the course of observation. The decreased serum creatinine levels observed in diabetic dogs is ascribed to the decreased muscle mass, which in turn was due to extreme emaciation ${ }^{11}$. The elevation of SALP level is presumably due to induction of canine-specific corticoidinduced alkaline phosphatase isoenzyme ${ }^{12}$. The increase in serum total cholesterol concentration is associated with altered lipid metabolism in diabetes ${ }^{11,12}$. The significance of thrombocytosis remains unknown. However, it is worthy to note that an increase in glucocorticoids is one of the causes of hyperglycemia ${ }^{12}$. Moreover, hypercortisolemia has been reported to be associated with thrombocytosis, which is due to increased production of thrombocytes in $\operatorname{dogs}^{13}$.

Different from the above-mentioned cases, four females were sporadically or only transiently diabetic without any signs of systemic involvement. These transiently diabetic cases are likely to be related to a condition of "impaired glucose tolerance"14. With respect to the diabetic episodes observed in this study, a close relationship between estrus and diabetes mellitus has been suggested in previous studies. For instance, an increased insulin requirement during estrus in dogs was described in a case report by Tischler ${ }^{15}$. In a recent textbook, diestrus has been pointed out to be a cause of insulin resistance and hyperglycemia in diabetic dogs and cats $^{9}$. Stockham and Scott have stated that progesterone released from the corpus luteum promotes the release of growth hormone, which in turn decreased the uptake of glucose by myocytes and adipocytes ${ }^{13}$. It is likely that the diabetic episodes recorded in the present study belong to the same category as "gestational diabetes mellitus" in humans ${ }^{14}$. 
Pathologic examination in our study revealed, as anticipated, major lesions in the pancreas, liver, kidneys, and eyes. Hypertrophy of the ductal epithelia of the pancreas as seen in the present study has already been described as the most remarkable change in the pancreas of alloxan-treated diabetic dogs ${ }^{8}$. Furthermore, similar changes, although only to a slight degree, have also been seen in surgically or anterior pituitary hormone induced diabetic dogs ${ }^{8}$. Cataractous changes similar to the typical picture of metabolic cataract ${ }^{16}$ were the sole major complication due to persistent hyperglycemia in the present study; however, no signs of histological or electro-retinographical evidence of retinopathy were observed. These findings are consistent with the results of previous researchers; Engelman et al. (1977), who observed alloxan treated and metabolically controlled dogs for 60 months, observed that cataracts develop only within the first two years ${ }^{4}$. Retinopathy, however, was found to be negligible even following many months after the onset of diabetes, but after about three years, retinopathy is observed more often with increasing duration of diabetes. In conclusion, the alloxan-induced beagle model of diabetes mellitus is useful for investigating the sequence of development by combined clinicopathological and histopathological examinations. Alloxan was not as diabetogenic to female beagles as male animals. Female animals with impaired glucose tolerance became transiently diabetic following diestrus. Secondary complications other than the cataracts did not occur easily in this model.

Acknowledgments: The authors gratefully acknowledge Ms. Kyoko Miura for her excellent assistance in manuscript preparation.

\section{References}

1. Rerup CC. Drugs producing diabetes through damage of the insulin secreting cells. Pharmacol Rev 1970; 22: 485-518.

2. Fischer LJ. Toxicity to the insulin-secreting beta cell. In: Comprehensive Toxicology, Boekleheide K, Chapin RE, Hoyer PB, and Harris C (eds), Amsterdam: Elsevier Science, Vol 10, 701-724, 1997.

3. Black HE, Rosenblum IY, and Capen CC. Chemically induced (Streptozotocin-Alloxan) diabetes mellitus in the dog: biochemical and ultrastructural studies. Am J Pathol 1980; 98: 295-305.

4. Engerman R, Bloodworth JMB Jr, and Nelson S. Relationship of microvascular disease in diabetes to metabolic control. Diabetes 1977; 26: 760-769.

5. Duncan JR, Prasse KW, and Mahaffey EA. Proteins, lipid, and carbohydrates. Urinary system. In: Veterinary Laboratory Medicine, clinical pathology, 3rd ed., Iowa: Iowa Sate Univ. Press, 162-183, 1994.

6. Watanabe D, Nakara H, Akagi K, Ishii T, Mizuguchi H, Nagashima Y, and Okaniwa A. Oral glucose tolerance test and determination of serum fructosamine level in beagle dogs. J Toxicol Sci 2004; 29: 33-36.

7. Engerman RN and Kramer JW. Dogs with induced or spontaneous diabetes as models for the study of human diabetes mellitus. Diabetes 1982; 31 (Suppl 1): 26-29.

8. Goldner MG and Gomori G. Alloxan diabetes in the dog. Endocrinology 1943; 33: 297-308.

9. Nelson RW. Disorders of the endocrine pancreas. In: Small animal internal medicine, Nelson RW and Couto CG (eds), Missouri: Mosby, 729-777, 2003.

10. Reush CE, Liehs MR, Hoyer M, and Vochezer R. Fructosamine: a new parameter for diagnosis and metabolic control in diabetic dogs and cats. J Vet Int Med 1993; 7: $177-182$.

11. Duncan JR, Prasse KW, and Mahaffey EA. Proteins, lipid, and carbohydrates. Urinary system. In: Veterinary Laboratory Medicine, clinical pathology, 3rd ed., Iowa: Iowa Sate Univ. Press, 112-129, 1994.

12. Meyer DJ and Harvey JW. Evaluation of hepatobiliary system and skeletal muscle and lipid disorders. In: Veterinary Laboratory Medicine, Interpretation \& Diagnosis, 2nd ed., Penn: WB Saunders, 157-186, 1998.

13. Stockham SL and Scott MA. Hyperglycemia. In: Fundamentals of Veterinary Clinical Pathology, Iowa: Iowa State Press, 487-506, 2002.

14. Sacks DB. Diabetes mellitus. In: Tietz Textbook of Clinical Chemistry, 3rd ed., Burtis CA and Aschwood ER (eds), Philadelphia: WB Saunders, 766-772, 1999.

15. Tischler SA. The effect of the estrous cycle on diabetes mellitus in the dog. J Amer Anim Hosp Assoc 1974; 10: $122-125$.

16. Gelatt KN. The canine lens. In: Veterinary Ophthalmology, 2nd ed., Gelatt KN (ed), Philadelphia and London: Lea \& Febiger, 429-460, 1991. 